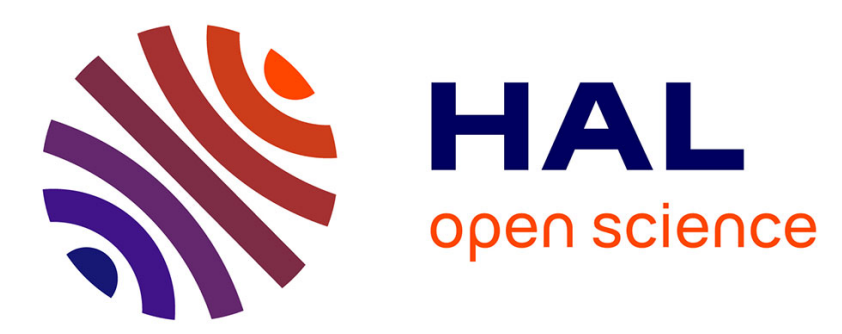

\title{
Robust Independent Component Analysis for Blind Source Separation and Extraction with Application in Electrocardiography
}

Vicente Zarzoso, Pierre Comon

\section{- To cite this version:}

Vicente Zarzoso, Pierre Comon. Robust Independent Component Analysis for Blind Source Separation and Extraction with Application in Electrocardiography. 30th Annual International Conference of the IEEE Engineering in Medicine and Biology Society, Aug 2008, Vancouver, Canada. pp.3344-3347. hal-00339616

\section{HAL Id: hal-00339616 https://hal.science/hal-00339616}

Submitted on 18 Nov 2008

HAL is a multi-disciplinary open access archive for the deposit and dissemination of scientific research documents, whether they are published or not. The documents may come from teaching and research institutions in France or abroad, or from public or private research centers.
L'archive ouverte pluridisciplinaire HAL, est destinée au dépôt et à la diffusion de documents scientifiques de niveau recherche, publiés ou non, émanant des établissements d'enseignement et de recherche français ou étrangers, des laboratoires publics ou privés. 


\title{
Robust Independent Component Analysis for Blind Source Separation and Extraction with Application in Electrocardiography
}

\author{
Vicente Zarzoso and Pierre Comon
}

\begin{abstract}
The problems of signal separation and signal extraction arise in a wide variety of applications in biomedical engineering and other areas. Under the source statistical independence assumption, these problems can be solved by independent component analysis (ICA) methods. A simple ICA technique, referred to as RobustICA, has recently been proposed that shows interesting features such as very fast convergence, local-extrema escaping capabilities and the possibility of avoiding prewhitening. The present contribution explains how RobustICA can easily be modified to target particular sources according to their impulsive character as measured by the kurtosis sign. This new feature makes it possible to extract the sources of interest only, or a subspace thereof, with the subsequent reduction in computational complexity and error accumulation. The performance of this modification is illustrated on signal recordings issued from electrocardiography.
\end{abstract}

\section{INTRODUCTION}

Extracting signals of interest from the observation of corrupted measurements is a fundamental signal processing problem arising in numerous applications including, but not limited to, biomedical engineering. Instances of this problem are found in electrocardiography. During pregnancy, the non-invasive measurement of the fetal heartbeat signal from maternal cutaneous recordings can provide more details about the child's well-being than currently employed techniques, mainly based on Doppler ultrasound heart-rate monitoring [1]. Unfortunately, the low-amplitude fetal cardiac signal is contaminated by the stronger maternal cardiac signal and other artifacts. In atrial fibrillation, the most prevalent arrhythmia encountered by physicians, the electrical signal generated in the atria contains useful information about the condition. Its dominant frequency is closely related to the refractory period of atrial myocardium cells, and thus to the stage and degree of organization of the disease; in particular, the lower the main frequency, the higher the probability of spontaneous cardioversion [2]. The non-invasive analysis of the atrial activity signal calls for the suppression of the ventricular complexes as well as other interference and noise contributing to the surface electrocardiogram (ECG).

Traditional approaches to signal extraction include frequency filtering and Wiener's optimal filtering [3]. However, in many practical scenarios like the above examples, the signal of interest and the interference often overlap in the frequency spectrum, and obtaining pure reference signals uncorrelated with the signal of interest may be a difficult task. The blind source separation (BSS) approach, introduced now

The authors are with the I3S Laboratory, UNSA/CNRS, Les Algorithmes, Euclide-B, BP 121, 2000 route des Lucioles, 06903 Sophia Antipolis Cedex, France; e-mail: $\{$ zarzoso, pcomon\}ei3s.unice.fr. over two decades ago, provides a more general framework. In its instantaneous linear mixture formulation, BSS assumes that the desired and interfering signals, so-called sources, may possibly have overlapping spectra and may all appear mixed together in each of the observations. The estimation of an appropriate extracting filters, and thus the estimation of the source waveforms from the observed mixtures, is achieved by recovering a known or assumed property of the sources. The assumption of statistical independence is very plausible in many applications like those recalled earlier. A mixture with arbitrary structure can be inverted regardless of the source spectra by imposing independence beyond second-order decorrelation, an operation referred to as independent component analysis (ICA) [4]. Most ICA algorithms proposed in the literature are based on non-Gaussianity measures such as the fourth-order statistics (cumulants), and can separate sources with non-zero kurtosis, regardless of its sign [4], [5], [6], [7]. Others can only separate sources with positive or negative kurtosis (respectively, super-Gaussian or sub-Gaussian sources), or with kurtoses verifying certain conditions [8]. The method of [7] exploits the prior knowledge of the source kurtosis signs to reduce the permutation ambiguity inherent to ICA. Basically, all the above methods aim at the joint separation of the source signals, although they may also be adapted to sequential extraction.

A simple technique called RobustICA has recently been presented in [9], [10] for sequential extraction of nonGaussian sources. The method is based on a general contrast function, the normalized kurtosis, which is maximized via a computationally efficient iterative search technique with an optimal step size. The present contribution shows how this method can easily be adapted to specifically target subGaussian or super-Gaussian sources. This feature enables the extraction of sources of interest when their Gaussianity character is known in advance, thus sparing a full separation of the observed mixture and the consequent unnecessary complexity and increased estimation error. The comparative performance of the modified algorithm is illustrated on a real ECG recording of atrial fibrillation.

\section{BLIND SOURCE SEPARATION AND EXTRACTION VIA ICA}

\section{A. Signal Model and Basic Assumptions}

In its instantaneous linear mixture formulation, BSS considers the signal model:

$$
\mathbf{x}(t)=\mathbf{H} \mathbf{s}(t)=\sum_{k=1}^{n} \mathbf{h}_{k} s_{k}(t)
$$


where vector $\mathbf{x}(t)=\left[x_{1}(t), x_{2}(t), \ldots, x_{m}(t)\right]^{\mathrm{T}}$ contains the observed signal mixtures, vector $\mathbf{s}(t)=$ $\left[s_{1}(t), s_{2}(t), \ldots, s_{n}(t)\right]^{\mathrm{T}}$ the unknown source signals, and $\mathbf{H}$ is the unknown $(m \times n)$ mixing matrix, assumed full column rank, whose coefficient $h_{i j}=[\mathbf{H}]_{i j}$ represents the contribution of source $j$ onto observation $i$. Symbol $(\cdot)^{\mathrm{T}}$ is the transpose operator. In what follows, time index $t$ will be dropped for convenience. Depending on the application, the sources and the mixture may take values in the real or in the complex field. Source separation is carried out by estimating a separating matrix $\mathbf{W}$ such that the separator output $\mathbf{y}=\mathbf{W}^{\mathrm{H}} \mathbf{x}$ contains an estimate of the sources, up to admissible scale and permutation indeterminacies, where symbol $(\cdot)^{\mathrm{H}}$ denotes the conjugate-transpose operator. Each column of $\mathbf{W}$ represents a spatial filter for the extraction of a single source, $y_{k}=\mathbf{w}_{k}^{\mathrm{H}} \mathbf{x}$.

Prewhitening. Principal component analysis (PCA) is probably the simplest approach to BSS under the independence assumption. This method exploits second-order statistics only and is generally unable to perform the separation. However, it reduces the problem to a set of whitened signals related to the sources through a unitary transformation: $\mathbf{z}=$ Qs. Matrix $\mathbf{Q}$ needs to be identified in a second step involving higher-order statistics. The prewhitening process introduces a bound on the achievable separation performance and may yield poor results in the presence of spatiallycorrelated noise with unknown covariance matrix.

\section{B. Contrast Functions, Cumulants and Non-Gaussianity}

The first thorough mathematical framework for BSS and ICA was established by Comon in [4]. A key concept in this formulation was the definition of contrast function, a functional $\Psi(\mathbf{y})$ in the distribution of the separator output quantifying the degree of separation. By virtue of three essential properties (invariance, domination and discrimination) characterizing a contrast, its optimization is achieved if and only if the sources are separated up to scale and permutation. Under the statistical independence assumption, the mutual information of the separator output is shown to constitute a valid contrast [4], and later found to be related to the maximum likelihood criterion [11], with the advantage of sparing the knowledge of the source distributions.

Approximations of information-theoretical contrasts lead to practical algorithms involving higher-order statistics (cumulants), easier to compute and to deal with. At fourth order, the minimization of mutual information is shown to be equivalent, under unitary transformations, to the maximization of the sum of square kurtoses of the separator output [4], [12]. The higher-order marginal cumulants of a Gaussian variable being null, this criterion is naturally connected to the maximization of non-Gaussianity of the separator output components. The Contrast Maximization (CoM2) method [4] maximizes this contrast iteratively by applying a planar Givens rotation to every signal pair until convergence, as in the Jacobi technique for matrix diagonalization.

\section{Sequential Extraction}

The above family of methods estimate all sources jointly or simultaneously. An alternative approach is to extract one source after another, a process known as sequential extraction or deflation [13]. In the real-valued case, it was proved in [13] that the maximization of criterion

$$
\left|\Psi_{\mathrm{KM}}(y)\right|, \quad \text { with } \Psi_{\mathrm{KM}}(y)=\frac{\kappa_{y}}{\sigma_{y}^{4}}
$$

is a valid contrast for the extraction of any source with nonzero kurtosis from model (1), with the extractor output given by $y=\mathbf{q}^{\mathrm{T}} \mathbf{z}$ and the unitary extracting vector $\mathbf{q}$ by the corresponding column of $\mathbf{Q}$. The kurtosis of a zero-mean random variable $y$ is defined as

$\kappa_{y}=\operatorname{cum}\left(y, y^{*}, y, y^{*}\right)=\mathrm{E}\left\{|y|^{4}\right\}-2 \mathrm{E}^{2}\left\{|y|^{2}\right\}-\left|\mathrm{E}\left\{y^{2}\right\}\right|^{2}$.

Symbol $\sigma_{y}^{4}$ denotes the square variance of the extractor output. After convergence of search algorithm, the contribution of the estimated source to the observations can be computed via the minimum mean square error solution to the linear regression problem $\mathbf{x}=\hat{\mathbf{h}} \hat{s}$, given by:

$$
\hat{\mathbf{h}}=\arg \max _{\mathbf{h}} \mathrm{E}\left\{\|\mathbf{x}-\mathbf{h} \hat{s}\|^{2}\right\}=\mathrm{E}\left\{\mathbf{x} \hat{s}^{*}\right\} / \mathrm{E}\left\{|\hat{s}|^{2}\right\} .
$$

The observations are then deflated as $\mathbf{x} \leftarrow(\mathbf{x}-\hat{\mathbf{h}} \hat{s})$ before re-initializing the algorithm in the search for the next source. This regression-based deflation is a procedure to avoid extracting the same source more than once.

In its original definition, the popular FastICA algorithm [6] aimed at the maximization of contrast (2). Some simplifications finally lead to the following update rule for extracting vector $\mathbf{q}$ :

$$
\mathbf{q}^{\prime}=\mathbf{q}-\frac{1}{3} \mathrm{E}\left\{\left(\mathbf{z}^{\mathrm{T}} \mathbf{q}\right)^{3} \mathbf{z}\right\}
$$

Vector $\mathbf{q}^{\prime}$ is then projected on the subspace orthogonal to the previously estimated extracting vectors, and normalized to keep the denominator of (2) constant. This approach to sequential extraction is called deflationary orthogonalization [6]. Equation (5) represents the FastICA method with cubic non-linearity, and is shown to have (asymptotically, for large sample size) global cubic convergence. Nevertheless, update rule (5) turns out to be a gradient-descent algorithm with constant step size [9]. Under analogous simplifications than its real-valued counterpart, the extension of FastICA with cubic non-linearity to the complex-valued scenario [14] neglects the non-circular part in the definition of kurtosis (3) and is thus restricted to circular source distributions.

\section{RobUSTICA}

\section{A. Optimal Step-Size Optimization}

Despite the simplifying assumptions (e.g,. prewhitening, real-valued sources and mixtures, circular complex sources etc.) made in previous works, criterion (2) is actually quite general. Indeed, it is a valid contrast for the extraction of a non-zero kurtosis source from mixture (1) whatever the type (real- or complex-valued) of sources and mixtures, and regardless of whether prewhitening has been carried 
out. More interestingly, this contrast can be maximized by an effective, computationally efficient search algorithm. Assuming an extractor output $y=\mathbf{w}^{\mathrm{H}} \mathbf{x}$, a quite natural update rule for the extracting vector $\mathbf{w}$ along an appropriate search direction $\mathbf{g}$ (e.g., the gradient) is given by

$$
\mathbf{w}^{\prime}=\mathbf{w}+\mu \mathbf{g}
$$

where the real-valued $\mu$ is the step size or adaption coefficient. In conventional search algorithms, $\mu$ is set to a constant or possibly time-varying value trying to balance a difficult trade-off between convergence speed and accuracy. Rather, we are interested in the value of $\mu$ that globally maximizes the normalized kurtosis contrast in the search direction:

$$
\mu_{\mathrm{opt}}=\arg \max _{\mu}\left|\Psi_{\mathrm{KM}}(y+\mu g)\right|
$$

where $g=\mathbf{g}^{\mathrm{H}} \mathbf{x}$. However, $\partial \Psi_{\mathrm{KM}}(y+\mu g) / \partial \mu$ is a rational function in $\mu$ with a fourth-degree polynomial as numerator. Hence, $\mu_{\mathrm{opt}}$ can be computed algebraically by finding the roots of this polynomial in the step size. Its coefficients, obtained in [9], [10], are functions of the observed data fourth-order statistics and the current values of the extracting vector and the search direction. The optimal step size is the root maximizing (7). The resulting method is referred to as RobustICA.

In the preliminary numerical results of [9], [10], RobustICA demonstrates a very fast convergence measured in terms of source extraction quality against number of operations. The optimal-step size update rule provides the method with some robustness to saddle points and spurious local extrema in the contrast function, which may appear when short data blocks are processed [15]. The generality of contrast (2) guarantees that RobustICA is able to separate real and complex (possibly non-circular) sources without any modification. In addition, the method does not require prewhitening, thus avoiding the associated performance limitations and increasing the robustness to spatially-coloured noise. Deflation is then carried out through linear regression, as in (4). Prewhitening can also be used, in conjunction with regression, or with deflationary orthogonalization as in FastICA.

\section{B. Extraction of Sources with Known Kurtosis Sign}

The method described above aims at maximizing the absolute normalized kurtosis, and is thus able to extract sources with positive or negative kurtosis. In many applications, some information may be known in advance about the source(s) of interest. For example, the atrial activity signal in atrial fibrillation, and especially in atrial flutter episodes, typically lies in the sub-Gaussian source subspace. In the separation of the fetal ECG from maternal skin recordings, the sources of interest, the fetal heartbeat signals, are usually impulsive and thus super-Gaussian. In these cases, separating the whole mixture would incur an unnecessary computational cost and, in the case of sequential extraction, an increased source estimation inaccuracy due to error accumulation through successive deflation stages. A more judicious alternative is extracting the desired type of sources alone.
RobustICA can easily be modified to deal with these situations by targeting a source with specific kurtosis sign $\varepsilon$. After computing the roots of the step-size polynomial, one simply needs to replace (7) by

$$
\mu_{\mathrm{opt}}=\arg \max _{\mu} \varepsilon \Psi_{\mathrm{KM}}(y+\mu g)
$$

as best root selection criterion. If no source exists with the required kurtosis sign, the algorithm may converge to a non-extracting local extrema, but will tend to produce components with maximal or minimal kurtosis from the remaining signal subspace when $\varepsilon=1$ or $\varepsilon=-1$, respectively. The algorithm can also be run by combining global line maximizations (7) and (8) for sources with known and unknown kurtosis sign, respectively, in any desired order. An implementation of the RobustICA algorithm including this feature is freely available in [16].

The method of [7] exploits the prior knowledge on the source kurtosis signs and, although originally designed for joint separation, it can easily be adapted to perform sequential extraction. Note, however, that it requires prewhitening.

\section{EXPERIMENTAL RESULTS}

Fig. 1(a) displays lead V1 of a standard 12-lead surface ECG recorded from an atrial fibrillation patient. ${ }^{1}$ Only $5 \mathrm{~s}$ are shown out of a $12 \mathrm{~s}$ segment sampled at $1 \mathrm{kHz}$. We apply the spatio-temporal BSS technique of [17] on these data. This technique consists of an initial ICA stage followed by a second-order refinement on the subspace spanned by the sources with the lowest kurtosis values (below a threshold empirically set at 1.5). This second stage, carried out by means of the SOBI algorithm [18], exploits the narrowband character of the atrial activity signal in order to improve its extraction from near-Gaussian sources. SOBI performs the joint approximate diagonalization of the whitened sensoroutput correlation matrices at different time lags. Fig. 1(b) shows the results provided by the ICA-based joint separation CoM2 method of [4]. FastICA with deflationary orthogonalization followed by SOBI estimates a very similar atrial contribution, as shown in Fig. 1(c). Finally, we run RobustICA [16] under the same working conditions as FastICA, but aiming for 6 sub-Gaussian sources only $(\varepsilon=-1)$. The application of SOBI on the obtained minimal-kurtosis source subspace provides the estimated atrial activity signal of Fig. 1(d), which seems a more accurate (less noisy) fit to the actual atrial activity observed in lead V1.

Fig. 2 displays the power spectra, estimated as in [17], of the signals in Fig. 1. The vertical axis of each plot is normalized with respect to the maximum amplitude of the corresponding spectrum in the $0-10 \mathrm{~Hz}$ frequency interval. Also shown is the frequency of the main peak, $f_{p}$, that all compared methods agree on estimating at $5.86 \mathrm{~Hz}$. The spectral concentration is computed as the percentage of signal power contained in the $0.82 f_{p}$ to $1.17 f_{p}$ frequency band [17]. This measure can be considered as an objective

\footnotetext{
${ }^{1}$ ECG recording courtesy of F. Castells and J. Millet from the Polytechnic University of Valencia, Spain.
} 


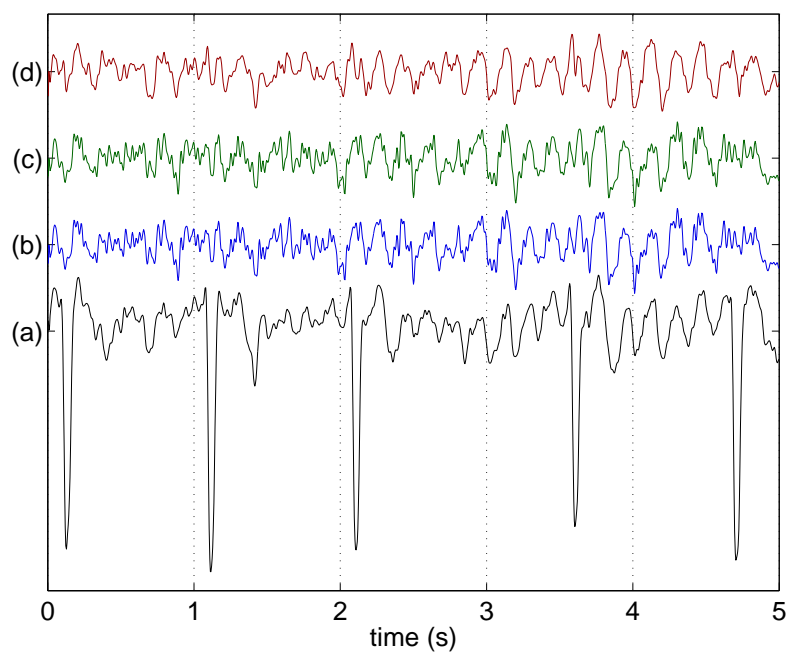

Fig. 1. (a) Lead V1 of an atrial fibrillation ECG. (b) Atrial activity contribution estimated by CoM2+SOBI. (c) Atrial activity contribution estimated by FastICA+SOBI. (d) Atrial activity contribution estimated by RobustICA+SOBI.

index of atrial activity extraction quality. According to this parameter, the proposed technique clearly outperforms the other methods.

Although not shown here due to lack of space, the application of RobustICA with $\varepsilon=1$ on a pregnant woman's surface ECG seems to allow the recovery of the fetal heartbeat subspace from two of the first five to six extracted sources.

\section{CONCLUSIONS}

The separation and extraction of independent nonGaussian sources can be carried out by algebraic exact line search optimization of the normalized kurtosis contrast, without simplifying assumptions. The resulting method is cost-effective, can escape spurious local extrema and does not require prewhitening. Prior knowledge on the kurtosis signs can easily be incorporated to aim for specific sources without separating the whole mixture. This leads to further savings in complexity and reductions in the accumulated estimation errors propagating through successive deflation stages. Results on real biomedical data have demonstrated the benefits of RobustICA, but are only illustrative; a more significant comparison should involve a complete recording database. In particular, the dimension of the minimal-kurtosis source subspace enabling the subsequent improvement in atrial activity estimation needs to be examined in more detail. The same algorithm can also deal with complex-valued possibly non-circular sources. Current research is exploring the exploitation of this interesting possibility in a biomedical context.

\section{REFERENCES}

[1] M. Peters, J. Crowe, J.-F. Piéri, et al., "Monitoring the fetal heart non-invasively: a review of methods," Journal of Perinatal Medicine, vol. 29, no. 5, pp. 408-416, Nov. 2001.

[2] A. Bollmann and F. Lombardi, "Electrocardiology of atrial fibrillation," IEEE Engineering in Medicine and Biology Magazine, vol. 25, no. 6, pp. 15-23, Nov./Dec. 2006.

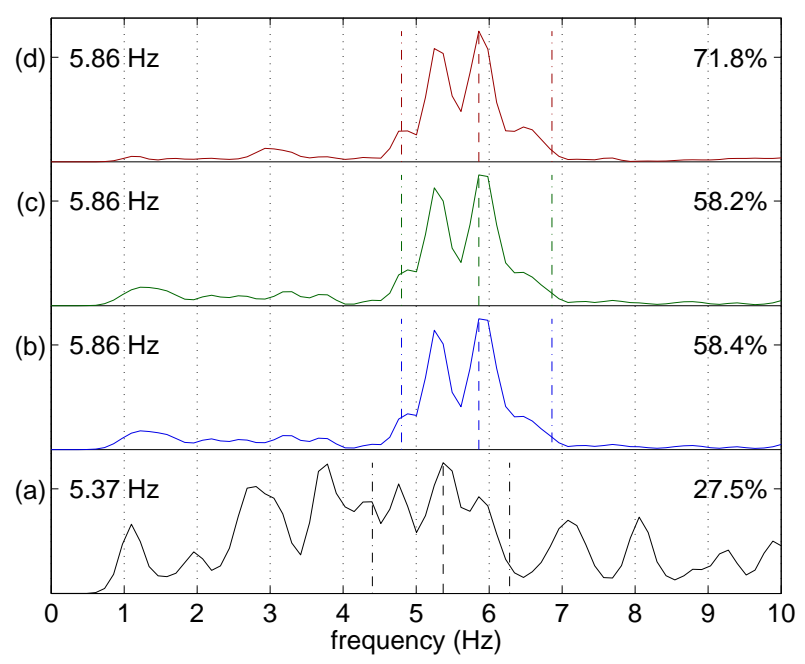

Fig. 2. Frequency spectra of the signals in Fig. 1. Values on the left: estimated main frequency, $f_{p}$ (also marked by the dashed lines). Values on the right: spectral concentration. The dash-dotted lines represent the bounds of the frequency band used to compute the spectral concentration.

[3] B. Widrow, J. R. Glover, J. M. McCool, and et al., "Adaptive noise cancelling: principles and applications," Proceedings of the IEEE, vol. 63, no. 12, pp. 1692-1716, Dec. 1975 .

[4] P. Comon, "Independent component analysis, a new concept?" Signal Processing, vol. 36, no. 3, pp. 287-314, Apr. 1994.

[5] J.-F. Cardoso and A. Souloumiac, "Blind beamforming for nonGaussian signals," IEE Proceedings- $F$, vol. 140 , no. 6, pp. 362-370, Dec. 1993.

[6] A. Hyvärinen, "Fast and robust fixed-point algorithms for independent component analysis," IEEE Transactions on Neural Networks, vol. 10, no. 3, pp. 626-634, May 1999.

[7] V. Zarzoso, R. Phlypo, and P. Comon, "A contrast for independent component analysis with priors on the source kurtosis signs," IEEE Signal Processing Letters, to appear.

[8] J.-F. Cardoso and B. H. Laheld, "Equivariant adaptive source separation," IEEE Transactions on Signal Processing, vol. 44, no. 12, pp. 3017-3030, Dec. 1996.

[9] V. Zarzoso, P. Comon, and M. Kallel, "How fast is FastICA?" in Proc. EUSIPCO-2006, XIV European Signal Processing Conference, Florence, Italy, Sept. 4-8, 2006.

[10] V. Zarzoso and P. Comon, "Comparative speed analysis of FastICA," in Proc. ICA-2007, 7th Intl. Conf. Independent Component Analysis and Signal Separation, London, UK, Sept. 9-12, 2007, pp. 293-300.

[11] J.-F. Cardoso, "Blind signal separation: statistical principles," Proceedings of the IEEE, vol. 86, no. 10, pp. 2009-2025, Oct. 1998.

[12] — - "Higher-order contrasts for independent component analysis," Neural Computation, vol. 11, pp. 157-192, 1999.

[13] N. Delfosse and P. Loubaton, "Adaptive blind separation of independent sources: a deflation approach," Signal Processing, vol. 45, no. 1, pp. 59-83, July 1995.

[14] E. Bingham and A. Hyvärinen, "A fast fixed-point algorithm for independent component analysis of complex valued signals," International Journal of Neural Systems, vol. 10, no. 1, pp. 1-8, Feb. 2000.

[15] P. Tichavsky, Z. Koldovsky, and E. Oja, "Performance analysis of the FastICA algorithm and Cramér-Rao bounds for linear independent component analysis," IEEE Transactions on Signal Processing, vol. 54, no. 4, pp. 1189-1203, Apr. 2006.

[16] "RobustICA package." [Online]. Available: www.i3s.unice.fr/ Zarzoso/robustica.html

[17] F. Castells, J. J. Rieta, J. Millet, and V. Zarzoso, "Spatiotemporal blind source separation approach to atrial activity estimation in atrial tachyarrhythmias," IEEE Transactions on Biomedical Engineering, vol. 52, no. 2, pp. 258-267, Feb. 2005.

[18] A. Belouchrani, K. Abed-Meraim, J.-F. Cardoso, and E. Moulines, "A blind source separation technique using second-order statistics," IEEE Transactions on Signal Processing, vol. 45, no. 2, pp. 434-444, Feb. 1997. 